\title{
A Derivative of the Gerasimov-Drell-Hearn Sum Rule
}

\author{
Vladimir Pascalutsa, $, 2, *$ Barry R. Holstein,, 3, , \\ ${ }^{1}$ Theory Group, JLab, 12000 Jefferson Ave, Newport News, VA 23606 \\ ${ }^{2}$ Department of Physics, College of William \& Mary, Williamsburg, VA 23188 \\ ${ }^{3}$ Department of Physics-LGRT, University of Massachusetts, Amherst, MA 01003
}

(Dated: August 4, 2018)

\begin{abstract}
We derive a sum rule which establishes a linear relation between a particle's anomalous magnetic moment and a quantity connected to the photoabsorption cross-section. This quantity cannot be measured directly. However, it can be computed within a given theory. As an example, we demonstrate validity of the sum rule in QED at tree level - the renowned Schwinger's correction to the anomalous magnetic moment is readily reproduced. In the case of the strong interactions, we also consider the calculation of the nucleon magnetic moment within chiral theories.
\end{abstract}

PACS numbers: 11.55.Hx, 13.40.Em, 25.20.Dc

*Electronic address: vlad@jlab.org

${ }^{\dagger}$ Electronic address: holstein@physics.umas.edu

${ }^{\ddagger}$ Electronic address: marcvdh@jlab.org 
The well-known Gerasimov-Drell-Hearn (GDH) sum rule (SR) [1],

$$
\frac{\pi \alpha}{M^{2} s} \kappa^{2}=\frac{1}{\pi} \int_{0}^{\infty} \frac{d \omega}{\omega} \Delta \sigma^{(\text {full })}(\omega),
$$

relates the anomalous magnetic moment (a.m.m.) [13] $\kappa$ of a particle with spin $s$ and mass $M$ to the integral of the difference of polarized total photoabsorption cross-sections:

$$
\Delta \sigma_{s}^{(\text {full })}(\omega)=\sigma_{1+s}^{(\text {full })}(\omega)-\sigma_{1-s}^{(\text {full })}(\omega)
$$

where $\sigma_{1 \pm s}^{(\text {full) }}$ are the photoabsorption cross sections for total helicity $(1 \pm s)$, and the superscript 'full' refers to the sum over all possible final states. Below we consider only the case of a spin- $1 / 2$ particle, hence $\Delta \sigma=\sigma_{3 / 2}-\sigma_{1 / 2}$. Furthermore, in Eq. (11), $\omega$ is the photon laboratory energy in the photoabsorption reaction, while $\alpha=e^{2} / 4 \pi \simeq 1 / 137$ is the fine structure constant.

The derivation of this SR is based on the general principles of analyticity (dispersion theory, with the assumed validity of an unsubtracted dispersion relation), unitarity (optical theorem), electromagnetic gauge-invariance, and crossing symmetry. Therefore any theory that satisfies these fundamental principles and which has the appropriate convergence property should be consistent with this SR, in the sense that the left-hand-side and the right-hand-side of Eq. (11), computed within that theory, must agree.

In QED, for instance, this consistency has long ago been verified, to lowest order in $\alpha$. Since in this case the lowest order contribution to the a.m.m. is of order $\alpha$, the lhs of the GDH SR Eq. (11) starts at $\mathcal{O}\left(\alpha^{3}\right)$. On the $r h s$, however, the tree-level contribution to the cross section difference $\Delta \sigma$ is non-vanishing at order $\alpha^{2}$. Hence, in order to satisfy the GDH $\mathrm{SR}$ at order $\alpha^{2}$ the lowest order integral in Eq. (11) must vanish :

$$
0=\int_{0}^{\infty} \frac{d \omega}{\omega} \Delta \sigma^{(\text {tree })}(\omega)
$$

which requires a careful cancellation between low and high frequency components of the photoabsorption cross sections. That this cancellation occurs precisely in QED and more generally in the standard electroweak theory has been verified by Altarelli, Cabibbo, and Maiani [3].

In order to check the consistency of the GDH SR at $\mathcal{O}\left(\alpha^{3}\right)$ in $\mathrm{QED}$, which corresponds with the familiar Schwinger correction to the a.m.m. $-\alpha / 2 \pi-$ one needs to consider all one-loop electromagnetic corrections to the photoabsorption cross sections on the rhs of Eq. (11). At this order, using the calculations of Tsai, deRaad, and Milton 4] the SR has only recently been verified by Dicus and Vega [5]. This calculation turns out to be rather involved, since one needs to compute the one-loop Compton amplitude, plus consider other inelastic processes, such as pair creation, then in the end perform the integral over photon energy. This allows the recovery of Schwinger's result which, of course, can be obtained much more easily by computing the one-loop vertex correction[6]. Thus, although one can obtain the same result for the a.m.m. by computing either the usual vertex correction or via the rhs of the GDH SR, the direct one-loop calculation is clearly much more straightforward and economical. 
In this note we shall present a new sum rule, for which the calculation of the dispersion integral over the photoabsorption cross section turns out to be simpler than the direct loop calculation - Schwinger's result can be obtained by integrating a tree-level cross section.

This sum rule can be derived from the GDH SR by introducing a "classical" value of the a.m.m., $\kappa_{0}$. At the field-theoretic level this implies that Dirac's Lagrangian of the spin-1/2 field acquires an explicit Pauli term:

$$
\mathcal{L}_{\text {Pauli }}=i \frac{e \kappa_{0}}{4 M} \bar{\psi} \sigma^{\mu \nu} \psi F_{\mu \nu}
$$

where $\sigma^{\mu \nu}=\frac{i}{2}\left[\gamma^{\mu}, \gamma^{\nu}\right]$ and $F_{\mu \nu}=\partial_{\mu} A_{\nu}-\partial_{\nu} A_{\mu}$ is the electromagnetic field tensor. The total value of the a.m.m. is then

$$
\kappa=\kappa_{0}+\delta \kappa,
$$

where by $\delta \kappa$ we denote all quantum (loop) corrections.

At this stage it is important to realize that in this theory with an explicit Pauli term the GDH SR is not valid, since there now exists a tree-level contribution to the Compton scattering amplitude which cannot be reproduced by a dispersion relation using the degrees of freedom included in the theory (e.g., photons and spin-1/2 fermions in case of QED). In fact, this tree-level contribution to the photoabsorption cross section, proportional to $\kappa_{0}^{2}$, corresponds with the d.o.f. which are integrated out of the theory, and can be represented as :

$$
\frac{2 \pi \alpha}{M^{2}} \kappa_{0}^{2}=\frac{1}{\pi} \int_{0}^{\infty} \frac{d \omega}{\omega} \Delta \sigma^{(\mathrm{he})}(\omega)
$$

where $\Delta \sigma^{(\text {he) }}$ is the photoabsorption cross sections with the 'high energy' d.o.f. integrated out of the theory. By subtracting Eq. (6) from Eq. (11), and expanding $\kappa$ as in Eq. (15), we obtain the sum rule :

$$
\frac{2 \pi \alpha}{M^{2}}\left\{(\delta \kappa)^{2}+2 \kappa_{0} \delta \kappa\right\}=\frac{1}{\pi} \int_{0}^{\infty} \frac{d \omega}{\omega} \Delta \sigma\left(\omega ; \kappa_{0}\right),
$$

where $\Delta \sigma \equiv \Delta \sigma^{(\text {full })}-\Delta \sigma^{(\text {he) }}$ corresponds to the photoabsorption cross-section that involves the d.o.f. included in the theory. Furthermore, we have indicated that the photoabsorption cross section depends explicitly on $\kappa_{0}$. Note that also $\delta \kappa$ will in general depend on $\kappa_{0}$, as the Pauli coupling appears in the loops.

By taking the limit to the theory with vanishing classical a.m.m., i.e. $\kappa_{0}=0$, one has $\delta \kappa \rightarrow \kappa$ and therefore Eq. (7) is nothing else than the GDH SR. We can however obtain a new sum rule by taking the first derivative with respect to $\kappa_{0}$ of both lhs and rhs of Eq. (77) and then let $\kappa_{0}=0$, which yields :

$$
\frac{4 \pi \alpha}{M^{2}} \kappa\left(1+\left[\frac{\partial}{\partial \kappa_{0}} \delta \kappa\right]_{\kappa_{0}=0}\right)=\frac{1}{\pi} \int_{0}^{\infty} \frac{d \omega}{\omega} \Delta \sigma_{1}(\omega),
$$

where we introduced the definition

$$
\Delta \sigma_{1}(\omega)=\left[\frac{\partial \Delta \sigma\left(\omega, \kappa_{0}\right)}{\partial \kappa_{0}}\right]_{\kappa_{0}=0}
$$


corresponding to the first derivative of the physical cross section with respect to $\kappa_{0}$. Note that in the derivation of Eq. (8) we did not rely on any perturbative expansion in coupling constants. This sum rule should then be valid non-pertubativelu as well as to any given order in perturbation theory. To lowest order in perturbation theory the term in the brackets on the left-hand side of Eq. (8) reduces to unity, yielding :

$$
\frac{4 \pi \alpha}{M^{2}} \kappa=\frac{1}{\pi} \int_{0}^{\infty} \frac{d \omega}{\omega} \Delta \sigma_{1}(\omega) .
$$

The striking feature of this sum rule is the linear relation between the a.m.m. and the photoabsorption cross section, in contrast to the GDH SR where the relation is quadratic. We wish to point out that, certainly, the cross-section quantity $\Delta \sigma_{1}$ is not an observable in the theory with $\kappa_{0}=0$. However, it is very clear how to determine it within a given theory.

As an example we consider QED, wherein to lowest order in $\alpha$ the photoabsorption crosssection is given by the Compton scattering tree graphs, see Fig. 1. Both the cross section and its $\kappa_{0}$ derivative at $\kappa_{0}=0$ can be straightforwardly computed to order $\alpha^{2}$ with the result:

$$
\begin{aligned}
\Delta \sigma(\omega) & =\frac{2 \pi \alpha^{2}}{M^{2} x}\left[2+\frac{2 x^{2}}{(1+2 x)^{2}}-\left(1+\frac{1}{x}\right) \ln (1+2 x)\right], \\
\Delta \sigma_{1}(\omega) & =\frac{2 \pi \alpha^{2}}{M^{2} x}\left[6-\frac{2 x}{(1+2 x)^{2}}-\left(2+\frac{3}{x}\right) \ln (1+2 x)\right],
\end{aligned}
$$

where $x=\omega / M$. As mentioned above, consistency of the GDH sum rule requires that the GDH integral of $\Delta \sigma$ vanishes [3] - cf. Eq. (3) - and this is easily verified for the form obtained in Eq. (11). As seen from Fig. 2, where we plot the corresponding integrand, there exists an exact cancellation between the low- and high-energy pieces of the cross section.

Evaluating the same type of integral for the derivative $\Delta \sigma_{1}$, shown by the dashed curve in Fig. 2, we find :

$$
\frac{1}{\pi} \int_{0}^{\infty} \frac{d \omega}{\omega} \Delta \sigma_{1}(\omega)=\frac{2 \alpha^{2}}{M^{2}} .
$$

Thus, employing the linearized GDH sum rule Eq. (10) we obtain $\kappa=\alpha / 2 \pi$-Schwinger's value! We wish to emphasize that this result has been obtained by calculating a tree-level cross section derivative with respect to the a.m.m., i.e., Eq. (12), and then performing the dispersion integral. That we obtain the same result as the usual calculation of a one-loop integral is a direct consequence of the general principles of analyticity, unitarity, gauge invariance, crossing symmetry, and convergence leading to an unsubtracted dispersion integral.

As a further example of how dispersion relations provide an economical method to evaluate one-loop corrections by calculating only tree level cross sections, we consider the theory of nucleons interacting with pions via pseudovector coupling:

$$
\mathcal{L}_{\pi N N}=\frac{g}{2 M} \bar{\psi} \gamma^{\mu} \gamma^{5} \tau^{a} \psi \partial_{\mu} \pi^{a}
$$

where $g$ is the pion-nucleon coupling constant, $\tau^{a}$ are isospin Pauli matrices, $\pi^{a}$ is the isovector pion field. To lowest order in $g$ the photoabsorption cross section in this theory 
is dominated by the single pion photoproduction graphs as displayed in Fig. 3. We find for the corresponding GDH cross sections:

$$
\begin{aligned}
\Delta \sigma^{\left(\pi^{0} p\right)} & =\frac{\pi C}{M^{2} x^{2}}\left[(2 \alpha \bar{s}+1-x) \ln \frac{\alpha+\lambda}{\alpha-\lambda}-2 \lambda[x(\alpha-2)+\bar{s}(\alpha+2)]\right], \\
\Delta \sigma^{\left(\pi^{+} n\right)} & =\frac{2 \pi C}{M^{2} x^{2}}\left[-\mu^{2} \ln \frac{\beta+\lambda}{\beta-\lambda}+2 \lambda(\bar{s} \beta-x \alpha)\right], \\
\Delta \sigma^{\left(\pi^{0} n\right)} & =0, \\
\Delta \sigma^{\left(\pi^{-} p\right)} & =\frac{2 \pi C}{M^{2} x^{2}}\left[-\mu^{2} \ln \frac{\beta+\lambda}{\beta-\lambda}+(2 \alpha \bar{s}-1-x) \ln \frac{\alpha+\lambda}{\alpha-\lambda}-2 \bar{s} \lambda\right],
\end{aligned}
$$

where $C=(e g / 4 \pi)^{2}, \mu=m_{\pi} / M, m_{\pi}$ is the pion mass, and

$$
\begin{aligned}
& s=M^{2}+2 M \omega, \bar{s}=s / M^{2}, \\
& \alpha=\left(s+M^{2}-m_{\pi}^{2}\right) / 2 s, \\
& \beta=\left(s-M^{2}+m_{\pi}^{2}\right) / 2 s=1-\alpha, \\
& \lambda=(1 / 2 s) \sqrt{s-\left(M+m_{\pi}\right)^{2}} \sqrt{s-\left(M-m_{\pi}\right)^{2}} .
\end{aligned}
$$

As in the case of QED, the anomalous magnetic moment corrections start at $\mathcal{O}\left(g^{2}\right)$, implying that the $l h s$ of the GDH SR begins at $\mathcal{O}\left(g^{4}\right)$. Since the tree-level cross sections are $\mathcal{O}\left(g^{2}\right)$, we must require that

$$
\int_{\omega_{\mathrm{th}}}^{\infty} \frac{d \omega}{\omega} \Delta \sigma^{(I)}(\omega)=0, \text { for } I=\pi^{0} p, \pi^{+} n, \pi^{0} n, \pi^{-} p,
$$

where $\omega_{\text {th }}=m_{\pi}\left(1+m_{\pi} / 2 M\right)$ is the threshold of the pion photoproduction reaction. This requirement is indeed verified for the expressions given in Eq. (15) - the consistency of GDH $\mathrm{SR}$ is maintained in this theory for each of the pion production channels.

Again, as in QED, there exists an intricate cancellation between the low- and high-energy component of these cross sections, $c f$. Fig. 4 (solid curves). Any attempted approximation to these cross-section, e.g., by means of semi-relativistic or chiral expansions could in principle violate this consistency with the GDH SR. This is indeed the case for the non-relativistic CGLN expressions [7], which are known to violate the SR [8]. It is plausible, however, that any such truncations can be performed in a fashion that maintains the consistency. For example, for the $\pi^{+}$production the CGLN expression can be supplemented by a term which is formally higher-order in $\omega / M$ to yield a cross-section:

$$
\Delta \sigma^{\left(\pi^{+} n\right)}=\frac{2 \pi C}{M^{2}}\left[-\frac{m_{\pi}^{2}}{\omega^{2}} \ln \left(\frac{\sqrt{\omega^{2}+m_{\pi}^{2}}+\omega}{\sqrt{\omega^{2}+m_{\pi}^{2}}-\omega}\right)+\frac{2 \omega}{M \bar{s}^{2}}\right],
$$

which still satisfies Eq. (17). It is interesting to observe that the addition of this new term (the second term in the square brackets) allows the semirelativistic approximation to have the same chiral limit as the full relativistic result:

$$
\lim _{m_{\pi} \rightarrow 0} \Delta \sigma^{\left(\pi^{+} n\right)}=\frac{2 \pi C}{M^{2}}\left[-\omega \delta(\omega)+\frac{2 \omega}{M \bar{s}^{2}}\right] .
$$


and from this expression it is particularly easy to see that the consistency is maintained in the chiral limit: $\int_{0}^{\infty} \frac{d \omega}{\omega} \lim _{m_{\pi} \rightarrow 0} \Delta \sigma^{(I)}=0$.

We now turn our attention to the linearized GDH sum rule. In this case we first introduce Pauli moments $\kappa_{0 p}$ and $\kappa_{0 n}$ for the proton and the neutron, respectively. The dependence of the cross-sections on these quantities can generally be presented as:

$$
\begin{aligned}
\Delta \sigma\left(\omega ; \kappa_{0 p}, \kappa_{0 n}\right) & =\Delta \sigma(\omega)+\kappa_{0 p} \Delta \sigma_{1 p}(\omega)+\kappa_{0 n} \Delta \sigma_{1 n}(\omega) \\
& +\kappa_{0 p}^{2} \Delta \sigma_{2 p}(\omega)+\kappa_{0 n}^{2} \Delta \sigma_{2 n}(\omega)+\kappa_{0 p} \kappa_{0 n} \Delta \sigma_{1 p 1 n}(\omega)+\ldots
\end{aligned}
$$

Furthermore, we introduce proton and neutron photoproduction cross sections $\Delta \sigma^{(p)}$ and $\Delta \sigma^{(n)}$ and express the corresponding GDH SRs, corrected by the $\kappa_{0}^{2}$ terms. Analogous to the QED case, we obtain :

(i) the GDH SRs:

$$
\frac{2 \pi \alpha}{M^{2}} \kappa_{p}^{2}=\frac{1}{\pi} \int_{\omega_{t h}}^{\infty} \frac{d \omega}{\omega} \Delta \sigma^{(p)}, \quad \frac{2 \pi \alpha}{M^{2}} \kappa_{n}^{2}=\frac{1}{\pi} \int_{\omega_{t h}}^{\infty} \frac{d \omega}{\omega} \Delta \sigma^{(n)}
$$

(ii) the linearized SRs (valid to leading order in the coupling $g$ ):

$$
\frac{4 \pi \alpha}{M^{2}} \kappa_{p}=\frac{1}{\pi} \int_{\omega_{t h}}^{\infty} \frac{d \omega}{\omega} \Delta \sigma_{1 p}^{(p)}, \quad \frac{4 \pi \alpha}{M^{2}} \kappa_{n}=\frac{1}{\pi} \int_{\omega_{t h}}^{\infty} \frac{d \omega}{\omega} \Delta \sigma_{1 n}^{(n)}
$$

(iii) the consistency conditions (valid to leading order in the coupling $g$ ):

$$
0=\frac{1}{\pi} \int_{\omega_{t h}}^{\infty} \frac{d \omega}{\omega} \Delta \sigma_{1 n}^{(p)}, \quad 0=\frac{1}{\pi} \int_{\omega_{t h}}^{\infty} \frac{d \omega}{\omega} \Delta \sigma_{1 p}^{(n)}
$$

The first derivatives of the cross-sections that enter in Eq. (21), to leading order in $g$, arise through the interference of Born graphs Fig. 3(a) with the graphs in Fig. 3(b) and we find:

$$
\begin{aligned}
\Delta \sigma_{1 p}^{(p)} \equiv \Delta \sigma_{1 p}^{\left(\pi^{0} p\right)}+\Delta \sigma_{1 p}^{\left(\pi^{+} n\right)} & =\frac{\pi C}{M^{2} x^{2}}\{2 x \lambda[4+(1-2 \alpha)(2+\bar{s}+2 x)]+2 \bar{s} \lambda(\alpha+2) \\
& \left.-\mu^{2} x \ln \frac{\beta+\lambda}{\beta-\lambda}+(2 \alpha \bar{s}+1-x) \ln \frac{\alpha+\lambda}{\alpha-\lambda}\right\} \\
\Delta \sigma_{1 n}^{(n)} \equiv \Delta \sigma_{1 n}^{\left(\pi^{0} n\right)}+\Delta \sigma_{1 n}^{\left(\pi^{-} p\right)} & =\frac{\pi C}{M^{2} x}\left\{2 \lambda(2+2 x-\bar{s})+\mu^{2} \ln \frac{\beta+\lambda}{\beta-\lambda}-\ln \frac{\alpha+\lambda}{\alpha-\lambda}\right\} \\
\Delta \sigma_{1 n}^{(p)} \equiv \Delta \sigma_{1 n}^{\left(\pi^{0} p\right)}+\Delta \sigma_{1 n}^{\left(\pi^{+} n\right)} & =\frac{2 \pi C}{M^{2} x^{2}}\left\{\ln \frac{\alpha+\lambda}{\alpha-\lambda}+2 \lambda(x \beta-\bar{s} \alpha)\right\} \\
\Delta \sigma_{1 p}^{(n)} \equiv \Delta \sigma_{1 p}^{\left(\pi^{0} n\right)}+\Delta \sigma_{1 p}^{\left(\pi^{-} p\right)} & =\frac{2 \pi C}{M^{2} x^{2}}\left\{(2 \bar{s} \alpha-x) \ln \frac{\alpha+\lambda}{\alpha-\lambda}+2 \lambda(x-2 \bar{s})\right\}
\end{aligned}
$$


Using the latter two expressions we easily verify the consistency conditions given in Eq. (21c) [14] while, employing the linearized SRs, we obtain:

$$
\begin{aligned}
\kappa_{p}^{(\text {loop })} & =\frac{M^{2}}{\pi e^{2}} \int_{\omega_{\text {th }}}^{\infty} \frac{d \omega}{\omega} \Delta \sigma_{1 p}^{(p)} \\
& =\frac{g^{2}}{(4 \pi)^{2}}\left\{1-\frac{\mu\left(4-11 \mu^{2}+3 \mu^{4}\right)}{\sqrt{1-\frac{1}{4} \mu^{2}}} \arccos \frac{\mu}{2}-6 \mu^{2}+2 \mu^{2}\left(-5+3 \mu^{2}\right) \ln \mu\right\}, \\
\kappa_{n}^{(\text {loop })} & =\frac{M^{2}}{\pi e^{2}} \int_{\omega_{\text {th }}}^{\infty} \frac{d \omega}{\omega} \Delta \sigma_{1 n}^{(n)}=\frac{-2 g^{2}}{(4 \pi)^{2}}\left\{2-\frac{\mu\left(2-\mu^{2}\right)}{\sqrt{1-\frac{1}{4} \mu^{2}}} \arccos \frac{\mu}{2}-2 \mu^{2} \ln \mu\right\} .
\end{aligned}
$$

Exactly the same result is found in the case of pseudoscalar pion-nucleon coupling and indeed Eq. (23) agrees with the long-known one-loop calculation done by using standard techniques [9]. It is also worth noting that the result given in Eq. (23) is not entirely in agreement with the chiral perturbation theory calculation of Ref. 10]. The discrepancy is apparently due to the fact that the "infrared regularized" loop amplitudes exploited in [10] do not satisfy the usual dispersion relations. Their analytic structure in the energy plane is somewhat more complicated since there are additional cuts due to explicit dependence on $\sqrt{s}$, cf. [11].

Finally, we would like to make an observation concerning the chiral behavior of the oneloop result for the nucleon a.m.m.. Expanding Eq. (23) around the chiral limit $\left(m_{\pi}=0\right)$, which incidentally corresponds here with the heavy-baryon expansion, we have

$$
\begin{aligned}
& \kappa_{p}^{\text {(loop) }}=\frac{g^{2}}{(4 \pi)^{2}}\left\{1-2 \pi \mu-2(2+5 \ln \mu) \mu^{2}+\frac{21 \pi}{4} \mu^{3}+O\left(\mu^{4}\right)\right\} \\
& \kappa_{n}^{\text {(loop })}=\frac{g^{2}}{(4 \pi)^{2}}\left\{-4+2 \pi \mu-2(1-2 \ln \mu) \mu^{2}-\frac{3 \pi}{4} \mu^{3}+O\left(\mu^{4}\right)\right\} .
\end{aligned}
$$

The term linear in pion mass (recall that $\mu=m_{\pi} / M$ ) is the well-known leading nonanalytic (LNA) correction. On the other hand, expanding the same expressions around the large $m_{\pi}$ limit we find

$$
\begin{aligned}
& \kappa_{p}^{(\text {loop })}=\frac{g^{2}}{(4 \pi)^{2}}(5-4 \ln \mu) \frac{1}{\mu^{2}}+O\left(\mu^{-4}\right) \\
& \kappa_{n}^{\text {(loop) }}=\frac{g^{2}}{(4 \pi)^{2}} 2(3-4 \ln \mu) \frac{1}{\mu^{2}}+O\left(\mu^{-4}\right) .
\end{aligned}
$$

What is intriguing here is that the one-loop correction to the nucleon a.m.m. for heavy quarks behaves as $1 / m_{\text {quark }}$ (where $m_{\text {quark }} \sim m_{\pi}^{2}$ ), precisely as expected from a constituent quark-model picture. Here this is a result of subtle cancellations in Eq. (23) taking place for large values of $m_{\pi}$. In contrast, the infrared regularization procedure [10] gives the result which exhibits pathological behavior with increasing pion mass and diverges for $m_{\pi}=2 M$.

Since the expressions in Eq. (23) have the correct large $m_{\pi}$ behavior they should be better suited for the chiral extrapolations of the lattice results than the usual heavy-baryon expansions or the "infrared-regularized" relativistic theory. This point is clearly demonstrated by Fig. 5, where we plot the $m_{\pi}$-dependence of the full [Eq. (23)], heavy-baryon, and 
infrared-regularization [10] leading order result for the magnetic moment of the proton and the neutron, in comparison to recent lattice data [12]. In presenting these results we have added a constant shift (counter-term $\kappa_{0}$ ) to the magnetic moment, i.e.,

$$
\begin{aligned}
& \mu_{p}=\left(1+\kappa_{0 p}+\kappa_{p}^{(\text {loop })}\right)(e / 2 M) \\
& \mu_{n}=\left(\kappa_{0 n}+\kappa_{n}^{(\text {loop })}\right)(e / 2 M)
\end{aligned}
$$

and fitted it to the known experimental value of the magnetic moment at the physical pion mass, $\mu_{p} \simeq 2.793$ and $\mu_{n} \simeq-1.913$, shown by the open diamonds in the figure. For the value of the $\pi N N$ coupling constant we have used $g^{2} / 4 \pi=13.5$. The $m_{\pi}$-dependence away off the physical point is then a prediction of the theory. The figure clearly shows that the SR results, shown by the dotted lines, is in a better agreement with the behavior obtained in lattice gauge simulations.

It is therefore convenient to use the SR results for the parametrization of lattice data. For example, we consider the following two-parameter form:

$$
\begin{aligned}
& \mu_{p}=\left(1+\frac{\tilde{\kappa}_{0 p}}{1+a_{p} m_{\pi}^{2}}+\kappa_{p}^{(\text {loop })}\right) \frac{e}{2 M}, \\
& \mu_{n}=\left(\frac{\tilde{\kappa}_{0 n}}{1+a_{n} m_{\pi}^{2}}+\kappa_{n}^{(\text {loop })}\right) \frac{e}{2 M}
\end{aligned}
$$

where $\tilde{\kappa}_{0 p}$ and $\tilde{\kappa}_{0 n}$ are fixed to reproduce the experimental magnetic moments at the physical $m_{\pi}$. The parameter $a$ can be fitted to lattice data. The solid curves in Fig. 5 represent the result of such a single parameter fit to the lattice data of Ref. [12] for the proton and neutron respectively, where $a_{p}=1.6 / M^{2}$ and $a_{n}=1.05 / M^{2}, M$ is the physical nucleon mass.

In conclusion, we have presented a new sum rule which in essence can be viewed as the first derivative of the well-known GDH sum rule w.r.t. the anomalous magnetic moment. The attractive feature of this new sum rule is that it established a linear relation between the a.m.m. and the cross section (in contrast to the GDH SR where the relation is quadratic), allowing an evaluation of loop corrections to the a.m.m. by computing a total cross section of a corresponding photoabsorption process to one loop lower than the desired result and then integrating it over energy. As an example, we reproduced in this way the celebrated Schwinger correction to the electron a.m.m., as well as considered the one pion-nucleon loop correction to the nucleon magnetic moment.

Of course, the results presented herein are not the end of such applications. Indeed, one can envision extension both to higher order calculations by use of one loop inputs and/or application to other sum rules, such as those for polarizabilities. However, we leave these as challenges for future work.

\section{Acknowledgments}

This work is supported in part by DOE grant no. DE-FG02-04ER41302 and contract DEAC05-84ER-40150 under which the Southeastern Universities Research Association (SURA) operates the Thomas Jefferson National Accelerator Facility. The work of BRH is also supported in part by NSF PHY-02-44801 and he would like to thank the JLab theory group 
for hospitality while this work was in progress.

[1] S. B. Gerasimov, Sov. J. Nucl. Phys. 2, 430 (1966) [Yad. Fiz. 2, 598 (1966)]; S. D. Drell and A. C. Hearn, Phys. Rev. Lett. 16, 908 (1966); M. Gell-Mann, M.L. Goldberger, and W.E. Thirring, Phys. Rev. 95, 1612 (1954).

[2] S. Weinberg, in Lectures on Elementary Particles and Quantum Field Theory, Vol. 1, Brandeis University Summer Institute 1970 (Deser, Grisaru and Pendleton, editors, M.I.T. Press, Cambridge, 1970); S. Ferrara, M. Porrati, and V.L. Telegdi, Phys. Rev. D 46, 3529 (1992).

[3] G. Altarelli, N. Cabibbo, and L. Maiani, Phys. Lett. B 40, 415 (1972).

[4] W. Tsai, L.L. deRaad, and K.A. Milton, Phys. Rev. D 6, 1428 (1972).

[5] D.A. Dicus and R. Vega, Phys. Lett. B 501, 44 (2001).

[6] See, e.g., J. D. Bjorken and S. D. Drell, Relativisitic Quantum Mechanics (Wiley, New York, 1964), Sect. 8.6.

[7] G. F. Chew, M. L. Goldberger, F. E. Low and Y. Nambu, Phys. Rev. 106, 1345 (1957).

[8] A. I. L'vov, Phys. Lett. B 304, 29 (1993).

[9] H. A. Bethe, and F. de Hoffman, Mesons and Fields, Vol. 2 (M.I.T. Press, Cambridge, 1970).

[10] B. Kubis and U. G. Meißner, Nucl. Phys. A 679, 698 (2001).

[11] T. Becher and H. Leutwyler, Eur. Phys. J. C 9, 643 (1999).

[12] J. M. Zanotti, S. Boinepalli, D. B. Leinweber, A. G. Williams and J. B. Zhang, Nucl. Phys. Proc. Suppl. 128, 233 (2004) arXiv:hep-lat/0401029.

[13] Here by the anomalous magnetic moment we understand $\kappa=(g-2) s$, i.e., the deviation of the gyromagnetic ratio $g$ from its natural value of 2 for any spin [2].

[14] We checked that these conditions are verified as well for the case of pseudoscalar $\pi N N$ coupling. 
(a)
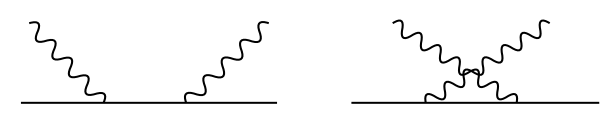

(b)
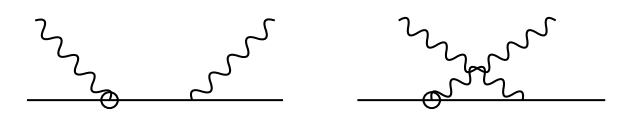

FIG. 1: Tree-level Compton scattering graphs. The circled vertex corresponds to the Pauli coupling.

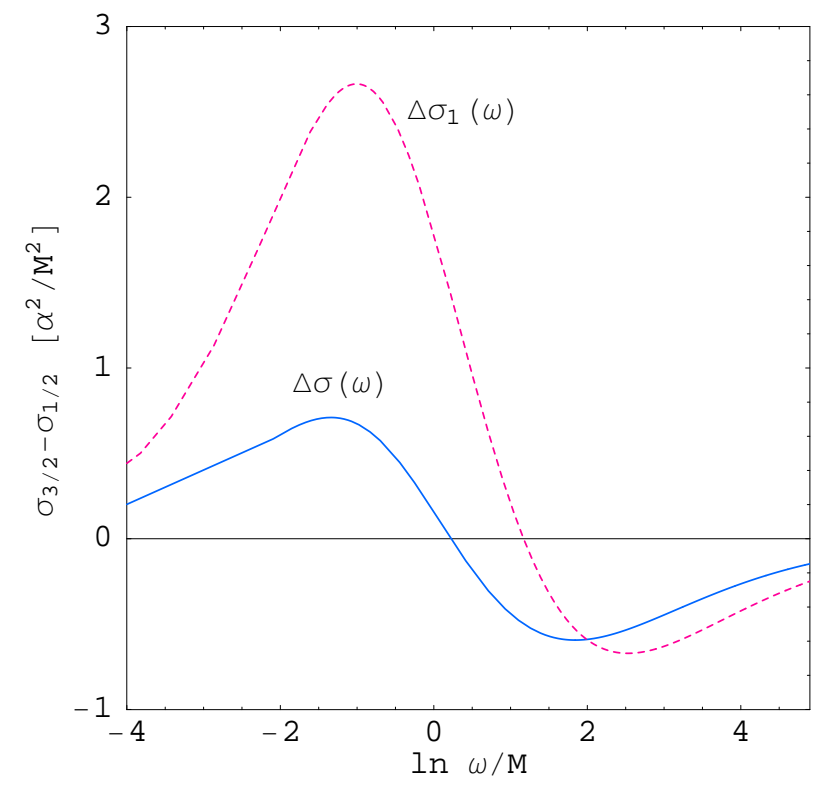

FIG. 2: GDH integrands for Compton scattering cross-sections (in units of $\alpha^{2} / M^{2}$ ), corresponding to Eqs. (11) and (12).

(a)
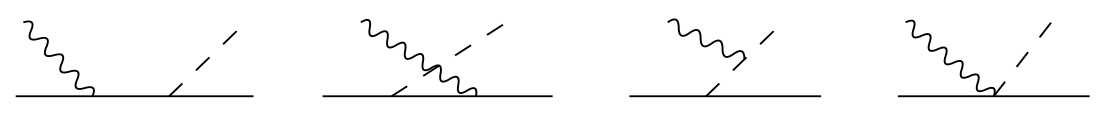

(b)
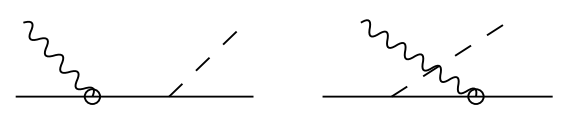

FIG. 3: Tree-level pion photoproduction graphs. The circled vertex corresponds to the Pauli coupling. 


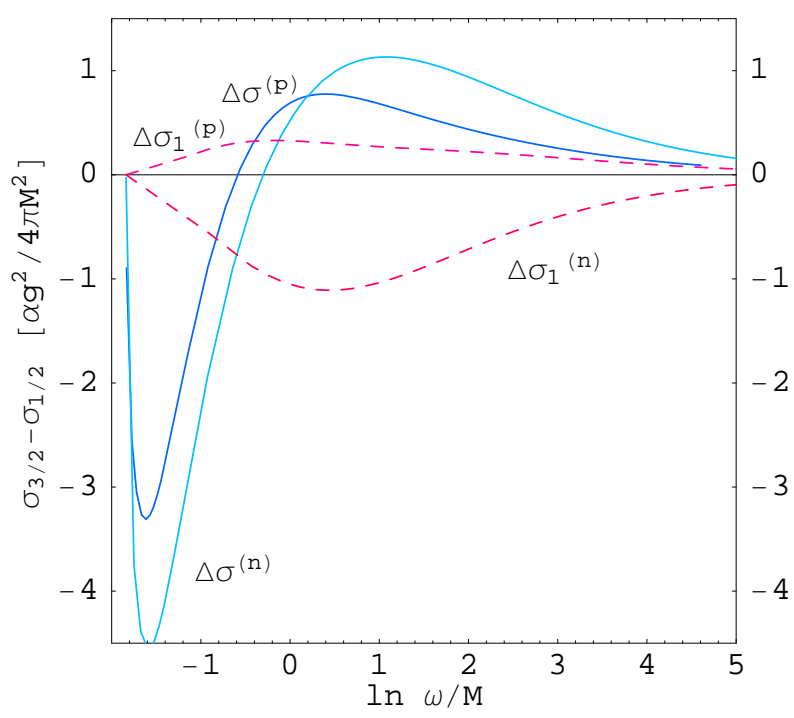

FIG. 4: GDH integrands (in units of $C / M^{2}$ ) for Born-level pion photoproduction cross sections, corresponding to Eqs. (15) and (22).
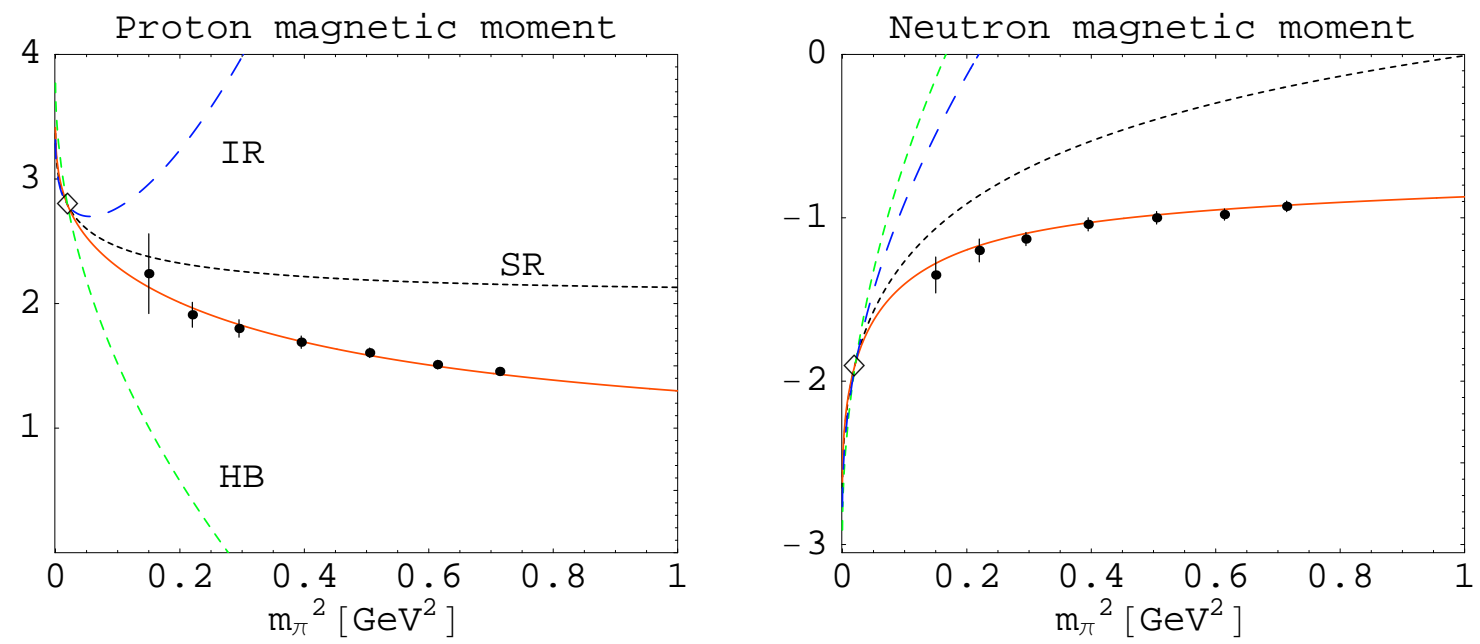

FIG. 5: Chiral behavior of the proton and neutron magnetic moments (in nucleon magnetons) to one loop compared with lattice data. "SR" (dotted lines) represents the full result given by Eqs. (23), "IR" (blue long-dashed lines) the infrared-regularized relativistic result, "HB" (green dashed lines) the LNA term in the heavy-baryon expansion Eq. (24). Red solid lines are the fit of the parametrization in Eq. (30) based on the SR result. Data points are results of lattice simulations [12]. The open diamonds represent the experimental values at the physical pion mass. 